\title{
Boltzmann kinetic equation for a strongly confined gas of hard spheres
}

\author{
J. Javier Brey, M. I. García de Soria, and P. Maynar \\ Física Teórica, Universidad de Sevilla, Apartado de Correos 1065, E-41080, Sevilla, Spain
}

(Received 19 June 2017; published 9 October 2017)

\begin{abstract}
A Boltzmann-like kinetic equation for a quasi-two-dimensional gas of hard spheres is derived. The system is confined between two parallel hard plates separated a distance between one and two particle diameters. An entropy Lyapunov function for the equation is identified. In addition to the usual Boltzmann expression, it contains a contribution associated to the confinement of the particles. The steady properties of the system agree with equilibrium statistical mechanics results. Equations describing the energy transfer between the degrees of freedom parallel and perpendicular to the confining plates are obtained for some simple initial configurations. The theoretical predictions are compared with molecular dynamics simulation data and a good agreement is found.
\end{abstract}

DOI: 10.1103/PhysRevE.96.042117

\section{INTRODUCTION}

The study of confined fluids has attracted a lot of attention since long time ago. Confinement is known to strongly affect the physical behavior of a fluid [1]. Most of the studies up to now have focused on equilibrium properties and, more specifically, on phase transitions and critical phenomena [2-5]. A geometry widely investigated is a system confined between two parallel hard plates [5,6]. In recent years many experiments have been performed in a system of macroscopic hard spheres confined between two large parallel plates separated a distance smaller than two grain diameters. This leads to an effective monolayer system, and it has been realized that the twodimensional dynamics of the projection of the spheres motion resembles that of a two-dimensional fluid, exhibiting also a series of phase transitions [7-9]. An important advantage of this system is that it can be easily implemented in the laboratory, but the price to be paid is that actual collisions between macroscopic spherical particles are inelastic, i.e., one has to consider a confined granular gas [10], which is by definition out of equilibrium. Actually, to keep the system fluidized, it is necessary to continuously inject energy into the system, e.g., by vibrating the hard walls.

The aim of this paper is to investigate the possibility of developing a macroscopic, hydrodynamiclike theory for confined systems with slit geometry. Attention will be restricted here to elastic, molecular systems, leaving for a further study the extension to granular, inelastic systems. Kinetic theory [11,12] and nonequilibrium statistical mechanics [13] provide the natural frameworks to investigate this issue. This implies to identify the relevant macroscopic fields, the form of the constitutive relations in some approximation (e.g., small gradients), and deriving the expressions of the involved transport coefficients. For extensive, nonconfined molecular systems, powerful theories have been developed to carry out the above program. In the case of dilute gases, a very robust and well-founded way of deriving hydrodynamics has been formulated starting from the Boltzmann kinetic equation [12,14]. Of course, the extension of the method to strongly confined systems requires as a first step to modify the Boltzmann equation, since the confinement limits the possible collisions occurring in the system and breaks down the isotropy of the fluid.

From equilibrium studies, it is known that the strong confinement between two plates affects the equilibrium properties of the fluid [6]. A nontrivial and relevant question is whether it also has some effects on the relaxation of the system towards the steady state, i.e., if an $H$ theorem, extension of the one formulated by Boltzmann, holds for confined systems and, if this is the case, what is the meaning of the new Lyapunov function.

Once the kinetic equation has been established and its steady solution has been determined, showing that it is always reached in the long time limit under appropriate boundary conditions, one can tackle the issue of the time evolution of the average, macroscopic properties of the system. The simplest ones are those which refer to global quantities under as homogeneous conditions as possible. In this paper, partial temperature parameters, associated with the kinetic energy carried out by the vertical and horizontal degrees of freedom are considered. Approximate evolution equations will be derived for them. Their solutions will be compared with molecular dynamics simulation results and a quite good agreement is found.

The remainder of this paper is organized as follows. In the next section, the Boltzmann kinetic equation for a system of hard spheres, confined between two hard parallel plates separated a distance smaller than two particle diameters, is derived. The evolution of the one-particle distribution function can be described by a kinetic equation, valid between the two plates, and some boundary conditions. The $H$ theorem and the approach to the stationary solution is discussed in Sec. III. It is shown that the stationary solution is consistent with results previously derived by means of density functional theory. Some of the results of the above sections have already been summarized, without proof, in Ref. [15]. There, it was also shown that the theoretical predictions for the steady density profile are in good agreement with molecular dynamics (MD) simulation results. In Sec. IV, the evolution equations for the temperature parameters associated with the vertical and horizontal degrees of freedom are derived for systems which are homogeneous in the horizontal plane. The results are validated by comparing with MD simulation data. Section V contains a few final comments and a summary of the conclusions. Finally, in the two appendices some details of the calculations carried out along the paper are reported.

\section{THE KINETIC EQUATION}

We consider a system of $N$ elastic hard spheres of mass $m$ and diameter $\sigma$, confined between two horizontal parallel hard 


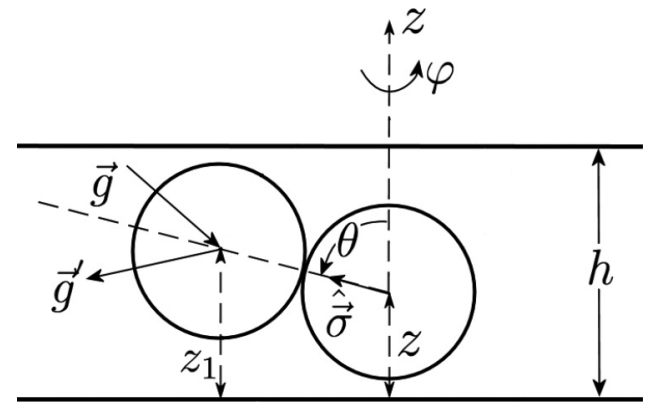

FIG. 1. Sketch of the collision between two particles in the quasitwo-dimensional system of hard spheres described in the main text. Particles are confined by two hard parallel walls, separated a distance $h$ between one and two particle diameters. Given the coordinate $z$ of one of the particles, there is a biunivocal correspondence between the coordinate $z_{1}$ of the other particle involved in the collision and the polar angle $\theta$.

plates separated a distance $h$, taking a value in the interval $\sigma<$ $h<2 \sigma$. Therefore, particles can never jump one over another. There is no external force acting on the particles other than the one associated with the hard walls. When two particles with velocities $\boldsymbol{v}$ and $\boldsymbol{v}_{1}$ collide, their velocities are instantaneously changed to the postcollisional velocities $\boldsymbol{v}^{\prime}$ and $\boldsymbol{v}_{1}^{\prime}$, respectively, given by

$$
\begin{aligned}
\boldsymbol{v}^{\prime} & \equiv b_{\boldsymbol{\sigma}} \boldsymbol{v}=\boldsymbol{v}+(\boldsymbol{g} \cdot \widehat{\boldsymbol{\sigma}}) \widehat{\boldsymbol{\sigma}}, \\
\boldsymbol{v}_{1}^{\prime} & \equiv b_{\boldsymbol{\sigma}} \boldsymbol{v}_{1}=\boldsymbol{v}_{1}-(\boldsymbol{g} \cdot \widehat{\boldsymbol{\sigma}}) \widehat{\boldsymbol{\sigma}},
\end{aligned}
$$

where $\boldsymbol{g} \equiv \boldsymbol{v}_{1}-\boldsymbol{v}$ is the relative velocity before the collision and $\widehat{\sigma}$ is the unit vector directed along the line joining the centers of the two particles at contact and pointing toward particle 1 . The above equations define the operator $b_{\sigma}$. The confinement of the system between the two plates implies restrictions over the possible directions of $\widehat{\sigma}$, as it is easily realized from Fig. 1, in which a sketch of a collision and the geometry of the system is presented, as well as the coordinates system to be used in the following. In particular, the $z$ axis is taken perpendicular to the hard plates, with its origin at one of them. It is assumed that in the dilute limit, the one-particle distribution function of the gas, $f(\boldsymbol{r}, \boldsymbol{v}, t)$, giving the density of particles at position $\boldsymbol{r}$ with velocity $\boldsymbol{v}$ at time $t$, obeys a Boltzmann-like kinetic equation in which the effects of the free motion of particles, of the collisions between particles, and of the collisions of the particles with the walls, can be treated independently [11-14],

$$
\frac{\partial f}{\partial t}+\boldsymbol{v} \cdot \frac{\partial f}{\partial \boldsymbol{r}}=J[\boldsymbol{r}, \boldsymbol{v} \mid f]+\bar{T}_{W} f .
$$

Here $J[\boldsymbol{r}, \boldsymbol{v} \mid f]$ denotes the contribution due to the collisions between particles while $\bar{T}_{W} f$ is the evolution term due to the interactions of the particles with the two horizontal walls. The latter are considered to be formally infinite, so that no boundaries parallel to the $z$ plane will be introduced. The explicit form of the two terms on the right-hand side of Eq. (3) will be discussed below.

To derive the expression of the collision term, a slight modification of the standard procedure used to obtain the
Boltzmann equation of a system of hard spheres will be used [11-14]. The term is decomposed into a gain contribution part $J_{+}$and a loss contribution part $J_{-}$,

$$
J[\boldsymbol{r}, \boldsymbol{v} \mid f]=J_{+}[\boldsymbol{r}, \boldsymbol{v} \mid f]+J_{-}[\boldsymbol{r}, \boldsymbol{v} \mid f] .
$$

The first term on the right-hand side is defined such that $J_{+} d \boldsymbol{r} d \boldsymbol{v} \delta t$ is the increase of $f d \boldsymbol{r} d \boldsymbol{v}$ due to collisions in the time interval between $t$ and $t+\delta t$. On the other hand, $J_{-} d \boldsymbol{r} d \boldsymbol{v} \delta t$ is the decrease of the same quantity in the same time interval, also due to collisions. To compute these quantities, it is necessary to take into account the confinement, since it limits the possible particle collisions occurring inside the system. As usual, it will be assumed that the one-particle distribution function does not vary over displacements of the order of the diameter $\sigma$ of the particles in a direction parallel to the plates. The same cannot be expected to hold in the $z$ direction, perpendicular to the walls, since the confinement imposed by the hard boundaries occurs over a distance smaller than $2 \sigma$, and the isotropy of the dynamics is clearly broken. Then, the number of particles with velocities in the interval $\left(\boldsymbol{v}_{1}, \boldsymbol{v}_{1}+d \boldsymbol{v}_{1}\right)$ colliding with a tagged particle of velocity $\boldsymbol{v}$ located in the volume element $d \boldsymbol{r}$ around $\boldsymbol{r}$ in the time interval between $t$ and $t+\delta t$, being the collision characterized by the unit vector $\widehat{\sigma}$ pointing inside the solid angle element $d \widehat{\boldsymbol{\sigma}}$, is

$$
\sigma^{2} \Theta(-\boldsymbol{g} \cdot \widehat{\boldsymbol{\sigma}})|\boldsymbol{g} \cdot \widehat{\boldsymbol{\sigma}}| f\left(\boldsymbol{r}+\boldsymbol{\sigma}_{z}, \boldsymbol{v}_{1}, t\right) d \widehat{\boldsymbol{\sigma}} d \boldsymbol{v}_{1} \delta t,
$$

where $\Theta$ is the Heaviside step function and

$$
\boldsymbol{\sigma}_{z} \equiv \sigma\left(\widehat{\boldsymbol{\sigma}} \cdot \boldsymbol{e}_{z}\right) \boldsymbol{e}_{z},
$$

with $\boldsymbol{e}_{z}$ being the unit vector in the positive direction of the $z$ axis. Since the number of particles with velocity between $v$ and $\boldsymbol{v}+d \boldsymbol{v}$ inside the volume element $d \boldsymbol{r}$ around $\boldsymbol{r}$ at time $t$ is $f(\boldsymbol{r}, \boldsymbol{v}, t) d \boldsymbol{r} d \boldsymbol{v}$, it follows that

$$
\begin{aligned}
J_{-}[\boldsymbol{r}, \boldsymbol{v} \mid f]= & \sigma^{2} \int d \boldsymbol{v}_{1} \int_{\Omega_{\sigma}(z)} d \widehat{\boldsymbol{\sigma}} \Theta(-\boldsymbol{g} \cdot \widehat{\boldsymbol{\sigma}})|\boldsymbol{g} \cdot \widehat{\boldsymbol{\sigma}}| \\
& \times f\left(\boldsymbol{r}+\boldsymbol{\sigma}_{z}, \boldsymbol{v}_{1}, t\right) f(\boldsymbol{r}, \boldsymbol{v}, t) .
\end{aligned}
$$

In the derivation of this expression, the molecular-chaos assumption or stossahlansatz has been employed, i.e., it has been assumed that the dynamical states of two particles involved in a collision are not correlated before the collision. Its justification in the present context is the same as for the derivation of the usual Boltzmann equation [11-14] and it will be not repeated here. The solid angle integration is restricted to the domain $\Omega_{\sigma}(z)$ compatible with the imposed confinement. From Fig. 1 it is seen that the restriction only concerns the value of $\widehat{\boldsymbol{\sigma}} \cdot \boldsymbol{e}_{z}$ and that the possible values depend on the coordinate $z$ of the tagged particle. The solid angle element $d \widehat{\sigma}$ can be expressed as

$$
d \widehat{\boldsymbol{\sigma}}=\sin \theta d \theta d \varphi,
$$

where $\theta$ is the polar angle measured with respect to the $z$ axis and $\varphi$ is the corresponding azimuth angle in the perpendicular plane. It is now convenient to make a change of variable from $\theta$ to $z_{1}$,

$$
z_{1}=z+\sigma \cos \theta
$$

The interval variation of $z_{1}$ is $\sigma / 2<z_{1}<h-\sigma / 2$, independently of the value of $z$. In the new variable, Eq. (7) becomes 


$$
\begin{aligned}
& (\boldsymbol{r} \equiv(x, y, z)) \\
& \begin{aligned}
J_{-}[\boldsymbol{r}, \boldsymbol{v} \mid f]= & \sigma \int d \boldsymbol{v}_{1} \int_{0}^{2 \pi} d \varphi \int_{\sigma / 2}^{h-\sigma / 2} d z_{1} \Theta(-\boldsymbol{g} \cdot \widehat{\boldsymbol{\sigma}}) \\
& \times|\boldsymbol{g} \cdot \widehat{\boldsymbol{\sigma}}| f\left(x, y, z_{1}, \boldsymbol{v}_{1}, t\right) f(\boldsymbol{r}, \boldsymbol{v}, t) .
\end{aligned}
\end{aligned}
$$

To compute $J_{+}$, collisions between particles with velocities in the intervals $\left(\boldsymbol{v}^{\prime}, \boldsymbol{v}^{\prime}+d \boldsymbol{v}^{\prime}\right)$ and $\left(\boldsymbol{v}_{1}^{\prime}, \boldsymbol{v}_{1}^{\prime}+d \boldsymbol{v}_{1}^{\prime}\right)$, respectively, are considered. These velocities are chosen such that after the collision defined by $\widehat{\boldsymbol{\sigma}}$, the first particle has a velocity between $\boldsymbol{v}$ and $\boldsymbol{v}+d \boldsymbol{v}$, i.e., the inverse collisions of the previous ones are being examined. Using the same kind of arguments as above, it is found that the number of such collisions occurring in the volume element $d \boldsymbol{r}$ around $\boldsymbol{r}$ in the time interval between $t$ and $t+d t$, and with a collision vector inside $d \widehat{\sigma}$ around $\widehat{\sigma}$ is given by

$$
\begin{aligned}
& \sigma^{2}\left|\boldsymbol{g}^{\prime} \cdot \widehat{\boldsymbol{\sigma}}\right| \Theta\left(-\boldsymbol{g}^{\prime} \cdot \widehat{\boldsymbol{\sigma}}\right) f\left(\boldsymbol{r}+\boldsymbol{\sigma}_{z}, \boldsymbol{v}_{1}^{\prime}, t\right) f\left(\boldsymbol{r}, \boldsymbol{v}^{\prime}, t\right) \\
& \quad \times d \widehat{\boldsymbol{\sigma}} d \boldsymbol{v}^{\prime} d \boldsymbol{v}_{1}^{\prime} d \boldsymbol{r} \delta t,
\end{aligned}
$$

where, of course, $\boldsymbol{g}^{\prime} \equiv \boldsymbol{v}_{1}^{\prime}-\boldsymbol{v}^{\prime}$. The Jacobian of the transformation between precollisonal and after-collisional velocities is unity, and the above expression is equivalent to

$$
\sigma^{2}|\boldsymbol{g} \cdot \widehat{\boldsymbol{\sigma}}| \Theta(\boldsymbol{g} \cdot \widehat{\boldsymbol{\sigma}}) f\left(\boldsymbol{r}+\boldsymbol{\sigma}_{z}, \boldsymbol{v}_{1}^{\prime}, t\right) f\left(\boldsymbol{r}, \boldsymbol{v}^{\prime}, t\right) d \widehat{\boldsymbol{\sigma}} d \boldsymbol{v} d \boldsymbol{v}_{1} d \boldsymbol{r} \delta t .
$$

Integrating over all the possible collisions, i.e., over the values of the velocity $\boldsymbol{v}_{1}$ and of the solid angle element $d \widehat{\boldsymbol{\sigma}}$, and transforming to the variable $z_{1}$, one easily gets

$$
\begin{aligned}
J_{+}[\boldsymbol{r}, \boldsymbol{v} \mid f]= & \sigma \int d \boldsymbol{v}_{1} \int_{0}^{2 \pi} d \varphi \int_{\sigma / 2}^{h-\sigma / 2} d z_{1} \Theta(\boldsymbol{g} \cdot \widehat{\boldsymbol{\sigma}})|\boldsymbol{g} \cdot \widehat{\boldsymbol{\sigma}}| \\
& \times f\left(x, y, z_{1}, \boldsymbol{v}_{1}^{\prime}, t\right) f\left(\boldsymbol{r}, \boldsymbol{v}^{\prime}, t\right)
\end{aligned}
$$

Combination of Eqs. (4), (10), and (13) readily leads to the expression of the collision term,

$$
\begin{aligned}
J[\boldsymbol{r}, \boldsymbol{v} \mid f]= & \sigma \int d \boldsymbol{v}_{1} \int_{0}^{2 \pi} d \varphi \int_{\sigma / 2}^{h-\sigma / 2} d z_{1}|\boldsymbol{g} \cdot \widehat{\boldsymbol{\sigma}}| \\
& \times \mid\left[\Theta(\boldsymbol{g} \cdot \widehat{\boldsymbol{\sigma}}) b_{\sigma}-\Theta(-\boldsymbol{g} \cdot \widehat{\boldsymbol{\sigma}})\right] f\left(x, y, z_{1}, \boldsymbol{v}_{1}, t\right) \\
& \times f(\boldsymbol{r}, \boldsymbol{v}, t) .
\end{aligned}
$$

To complete the kinetic equation, the walls contribution $\bar{T}_{W} f$ must be specified. Here, the simplest case of elastic walls at rest will be addressed. For this choice, application of the general ideas developed, for instance, in Refs. [11] and [14], gives

$$
\begin{gathered}
\bar{T}_{W}=\bar{T}_{W}^{(0)}+\bar{T}_{W}^{(h)}, \\
\bar{T}_{W}^{(0)} f(\boldsymbol{r}, \boldsymbol{v}, t)=\delta\left(z-\frac{\sigma}{2}\right) v_{z}\left[\Theta\left(v_{z}\right) f\left(\boldsymbol{r}, \boldsymbol{v}^{(*)}, t\right)\right. \\
\left.+\Theta\left(-v_{z}\right) f(\boldsymbol{r}, \boldsymbol{v}, t)\right], \\
\bar{T}_{W}^{(h)} f(\boldsymbol{r}, \boldsymbol{v}, t)=-\delta\left(z+\frac{\sigma}{2}-h\right) v_{z}\left[\Theta\left(-v_{z}\right) f\left(\boldsymbol{r}, \boldsymbol{v}^{(*)}, t\right)\right. \\
\left.+\Theta\left(v_{z}\right) f(\boldsymbol{r}, \boldsymbol{v}, t)\right] .
\end{gathered}
$$

In the above expressions, $\boldsymbol{v}^{(*)}$ denotes the precollisional value of the velocity such that after a collision with the wall, the particle acquires a velocity $\boldsymbol{v}$,

$$
\boldsymbol{v}^{(*)}=\boldsymbol{v}-2 v_{z} \boldsymbol{e}_{z} .
$$

In Appendix A a short overview of the derivation of Eqs. (16) and (17) is presented.

The dynamics defined by the kinetic Eq. (3) guarantees that the number of particles inside the system is conserved since particles can not leave or enter the system. Mathematically, if the initial one-particle distribution function vanishes outside the system, this property is kept in time by the kinetic equation. In the following, attention will be naturally restricted to that kind of initial conditions. Let us consider

$$
f(\boldsymbol{r}, \boldsymbol{v}, t)=W(z) \tilde{f}(\boldsymbol{r}, \boldsymbol{v}, t),
$$

where $W(z)$ is a characteristic function that vanishes if the sphere whose center is located at $\boldsymbol{r}$ has a part outside the system and it is unity if the complete particle is inside the system. More explicitly,

$$
W(z)=\Theta\left(z-\frac{\sigma}{2}\right) \Theta\left(h-\frac{\sigma}{2}-z\right) .
$$

It is assumed, without loss of generality, that $\tilde{f}$ and its derivatives are continuous functions of $\boldsymbol{r}$ as it approaches the walls from inside the system. Then, Eq. (3) can be decomposed into three separated equations, one containing only terms that are regular at both boundaries, another containing only terms which are singular at $z=\sigma / 2$, and a last equation with all the terms singular at $z=h-\sigma / 2$. This happens because

$$
\boldsymbol{v} \cdot \frac{\partial f}{\partial \boldsymbol{r}}=W(z) \boldsymbol{v} \cdot \frac{\partial \widetilde{f}}{\partial \boldsymbol{r}}+\tilde{f} v_{z} \frac{\partial W(z)}{\partial z} .
$$

The regular equation is

$$
W(z) \frac{\partial \tilde{f}}{\partial t}+W(z) \boldsymbol{v} \cdot \frac{\partial \tilde{f}}{\partial \boldsymbol{r}}=W(z) J[\boldsymbol{r}, \boldsymbol{v} \mid \tilde{f}],
$$

the equation with the terms singular at $z=\sigma / 2$ reads

$$
\Theta\left(v_{z}\right) \tilde{f}(\boldsymbol{r}, \boldsymbol{v}, t) \delta\left(z-\frac{\sigma}{2}\right)=\Theta\left(v_{z}\right) \tilde{f}\left(\boldsymbol{r}, \boldsymbol{v}^{*}, t\right) \delta\left(z-\frac{\sigma}{2}\right),
$$

and the equation with all the terms singular at $z=h-\sigma / 2$,

$$
\begin{aligned}
\Theta & \left(-v_{z}\right) \tilde{f}(\boldsymbol{r}, \boldsymbol{v}, t) \delta\left(z-h+\frac{\sigma}{2}\right) \\
& =\Theta\left(-v_{z}\right) \tilde{f}\left(\boldsymbol{r}, \boldsymbol{v}^{*}, t\right) \delta\left(z-h+\frac{\sigma}{2}\right) .
\end{aligned}
$$

Both singular equations, Eqs. (23) and (24), can be considered as boundary conditions to the Boltzmann Eq. (22), where the factor $W(z)$ can be committed if the equation is restricted to hold only inside the system, where $W(z)=1$. The physical interpretation of the boundary conditions is simple; they express the conservation of the flux of particles at the walls. In the following, and for the sake of simplicity, the tilde on the one-particle distribution function will be also omitted, so that the Boltzmann equation inside the system takes the form

$$
\frac{\partial f}{\partial t}+\boldsymbol{v} \cdot \frac{\partial f}{\partial \boldsymbol{r}}=J[\boldsymbol{r}, \boldsymbol{v} \mid f] .
$$

Other kind of hard boundary conditions, such as those associated with thermal walls, can be analyzed in a similar way $[11,14]$. 


\section{THE $H$ THEOREM AND THE EQUILIBRIUM DISTRIBUTION}

Let us define the following functional of $f$ :

$$
H(t) \equiv H^{k}(t)+H^{c}(t)
$$

where

$$
H^{k}(t) \equiv \int d \boldsymbol{r} \int d \boldsymbol{v} f(\boldsymbol{r}, \boldsymbol{v}, t)[\ln f(\boldsymbol{r}, \boldsymbol{v}, t)-1]
$$

and

$$
H^{c}(t) \equiv \frac{1}{2} \int d \boldsymbol{r} \int d \boldsymbol{r}_{1} n(\boldsymbol{r}, t) n\left(\boldsymbol{r}_{1}, t\right) \Theta\left(\sigma-\left|\boldsymbol{r}_{1}-\boldsymbol{r}\right|\right),
$$

$\boldsymbol{r}_{1} \equiv\left(x, y, z_{1}\right)$. In the last expression, $n(\boldsymbol{r}, t)$ is the number of particles density field, defined as

$$
n(\boldsymbol{r}, t) \equiv \int d \boldsymbol{v} f(\boldsymbol{r}, \boldsymbol{v}, t)
$$

The term $H^{k}$ is the same as considered in the celebrated $H$ theorem of the usual Boltzmann equation for a dilute gas [11-14], and it will be referred to as the "kinetic part" of $H$, while $H^{c}$ is suggested by the form of the equilibrium entropy of a gas of hard spheres in the approximation of the second viral coefficient $[15,16]$, and it will called "configurational part" of $H$, since it is associated with the confinement of the system between the two plates. The expression of $H^{c}$ can also be justified starting from a local equilibrium approximation for the $N$ particle distribution $[17,18]$. Consider first the time derivative of the kinetic part,

$$
\frac{\partial H^{k}(t)}{\partial t}=\int d \boldsymbol{r} \int d \boldsymbol{v}[\ln f(\boldsymbol{r}, \boldsymbol{v}, t)] \frac{\partial f(\boldsymbol{r}, \boldsymbol{v}, t)}{\partial t}
$$

The free flow term in the kinetic Eq. (25) leads to a vanishing contribution when substituted in the above equation. This is easily verified by using the Gauss theorem, and taking into account that (1) the flux of any property through the hard plates is trivially zero, given that they are elastic and at rest, and (2) the same happens in the $x$ and $y$ directions if one formally considers, for instance, periodic boundary conditions, so that the surface integral over the boundaries parallel to the $z$ axis also vanishes. Then, Eq. (30) reduces to

$$
\frac{\partial H^{k}(t)}{\partial t}=\int d \boldsymbol{r} \int d \boldsymbol{v} \ln f(\boldsymbol{r}, \boldsymbol{v}, t) J[\boldsymbol{r}, \boldsymbol{v} \mid f]
$$

Next, the general property

$$
\begin{aligned}
\int d \boldsymbol{v} \xi(\boldsymbol{v}) J[\boldsymbol{r}, \boldsymbol{v} \mid f]= & \sigma \int_{\sigma / 2}^{h-\sigma / 2} d z_{1} \int d \boldsymbol{v} \int d \boldsymbol{v}_{1} \int_{0}^{2 \pi} d \varphi \\
& \times\left[\xi\left(\boldsymbol{v}^{\prime}\right)-\xi(\boldsymbol{v})\right]|\boldsymbol{g} \cdot \widehat{\boldsymbol{\sigma}}| \Theta(-\boldsymbol{g} \cdot \widehat{\boldsymbol{\sigma}}) \\
& \times f\left(\boldsymbol{r}_{1}, \boldsymbol{v}_{1}, t\right) f(\boldsymbol{r}, \boldsymbol{v}, t),
\end{aligned}
$$

valid for arbitrary $\xi(v)$, is used to rewrite Eq. (31) as

$$
\begin{aligned}
\frac{\partial H^{k}(t)}{\partial t}= & \sigma \int d x \int d y \int_{\sigma / 2}^{h-\sigma / 2} d z \int_{\sigma / 2}^{h-\sigma / 2} d z_{1} \int d \boldsymbol{v} \int d \boldsymbol{v}_{1} \int_{0}^{2 \pi} d \varphi \\
& \times \ln \frac{f\left(\boldsymbol{r}, \boldsymbol{v}^{\prime}, t\right)}{f(\boldsymbol{r}, \boldsymbol{v}, t)}|\boldsymbol{g} \cdot \widehat{\boldsymbol{\sigma}}| \Theta(-\boldsymbol{g} \cdot \widehat{\boldsymbol{\sigma}}) f\left(\boldsymbol{r}_{1}, \boldsymbol{v}_{1}, t\right) f(\boldsymbol{r}, \boldsymbol{v}, t) .
\end{aligned}
$$

In the right-hand side of this expression, the variables $\left(z_{1}, \boldsymbol{v}_{1}\right)$ are interchanged with $(z, \boldsymbol{v})$ and, at the same time, $\varphi$ is changed into $\varphi+\pi$. This implies changing $\widehat{\sigma}$ into $-\widehat{\sigma}$. Then taking half the sum of the resulting expression with Eq. (33), it is obtained

$$
\begin{aligned}
\frac{\partial H^{k}(t)}{\partial t}= & \frac{\sigma}{2} \int d x \int d y \int_{\sigma / 2}^{h-\sigma / 2} d z \int_{\sigma / 2}^{h-\sigma / 2} d z_{1} \int d \boldsymbol{v} \int d \boldsymbol{v}_{1} \int_{0}^{2 \pi} d \varphi \\
& \times \ln \frac{f\left(\boldsymbol{r}, \boldsymbol{v}^{\prime}, t\right) f\left(\boldsymbol{r}_{1}, \boldsymbol{v}_{1}^{\prime}, t\right)}{f(\boldsymbol{r}, \boldsymbol{v}, t) f\left(\boldsymbol{r}_{1}, \boldsymbol{v}_{1}, t\right)}|\boldsymbol{g} \cdot \widehat{\boldsymbol{\sigma}}| \Theta(-\boldsymbol{g} \cdot \widehat{\boldsymbol{\sigma}}) f\left(\boldsymbol{r}_{1}, \boldsymbol{v}_{1}, t\right) f(\boldsymbol{r}, \boldsymbol{v}, t) .
\end{aligned}
$$

At this point, the inequality $x(\ln y-\ln x) \leqslant y-x$, valid for real $x, y>0$, will be employed. The equality sign only holds for $x=y$. In this way, it is obtained

$$
\begin{aligned}
\frac{\partial H^{k}(t)}{\partial t} \leqslant & \frac{\sigma}{2} \int d x \int d y \int_{\sigma / 2}^{h-\sigma / 2} d z \int_{\sigma / 2}^{h-\sigma / 2} d z_{1} \int d \boldsymbol{v} \int d \boldsymbol{v}_{1} \int_{0}^{2 \pi} d \varphi \\
& \times|\boldsymbol{g} \cdot \widehat{\boldsymbol{\sigma}}| \Theta(-\boldsymbol{g} \cdot \widehat{\boldsymbol{\sigma}})\left[f\left(\boldsymbol{r}, \boldsymbol{v}^{\prime}, t\right) f\left(\boldsymbol{r}_{1}, \boldsymbol{v}_{1}^{\prime}, t\right)-f(\boldsymbol{r}, \boldsymbol{v}, t) f\left(\boldsymbol{r}_{1}, \boldsymbol{v}_{1}, t\right)\right] .
\end{aligned}
$$

To proceed, the exchange of velocities $\left(\boldsymbol{v}^{\prime}, \boldsymbol{v}_{1}^{\prime}\right) \rightleftharpoons\left(\boldsymbol{v}, \boldsymbol{v}_{1}\right)$ is performed in the first integral on the right hand side, and it is taken into account that the only component of $\widehat{\sigma}$ giving a nonvanishing contribution is $\widehat{\sigma}_{z}=\cos \theta$. This leads to

$$
\frac{\partial H^{k}(t)}{\partial t} \leqslant \pi \int d x \int d y \int_{\sigma / 2}^{h-\sigma / 2} d z \int_{\sigma / 2}^{h-\sigma / 2} d z_{1}\left(z-z_{1}\right) n\left(\boldsymbol{r}_{1}, t\right) n(\boldsymbol{r}, t)\left[u_{z}\left(\boldsymbol{r}_{1}, t\right)-u_{z}(\boldsymbol{r}, t)\right],
$$

where the local macroscopic velocity field $\boldsymbol{u}(\boldsymbol{r}, t)$ has been defined as

$$
n(\boldsymbol{r}, t) \boldsymbol{u}(\boldsymbol{r}, t) \equiv \int d \boldsymbol{v} \boldsymbol{v} f(\boldsymbol{r}, \boldsymbol{v}, t) .
$$


Finally, the expression of $\cos \theta$ given in Eq. (9) is substituted, and afterwards the coordinates $z$ and $z_{1}$ are exchanged in one of the two terms on the right-hand side to arrive to the formula

$$
\begin{gathered}
\frac{\partial}{\partial t} H^{k} \leqslant I(t), \\
I(t) \equiv 2 \pi \int d x \int d y \int_{\sigma / 2}^{h-\sigma / 2} d z \int_{\sigma / 2}^{h-\sigma / 2} d z_{1} n(\boldsymbol{r}, t) n\left(\boldsymbol{r}_{1}, t\right)\left(z_{1}-z\right) u_{z}\left(\boldsymbol{r}_{1}, t\right) .
\end{gathered}
$$

To compute the time evolution of the confinement contribution to $H(t)$ defined in Eq. (28), it is convenient to use for $\boldsymbol{r}_{1}$ the coordinates $z_{1}, \zeta=\left|\boldsymbol{r}-\boldsymbol{r}_{1}\right|$, and $\varphi$. It is $d \boldsymbol{r}_{1}=\zeta d z_{1} d \zeta d \varphi$, so that from Eq. (28) it is easily obtained

$$
H^{c}(t)=\pi \int d x \int d y \int_{\sigma / 2}^{h-\sigma / 2} d z \int_{\sigma / 2}^{h-\sigma / 2} d z_{1} \int_{\left|z-z_{1}\right|}^{\infty} d \zeta n(\boldsymbol{r}, t) n\left(\boldsymbol{r}_{1}, t\right) \zeta \Theta(\sigma-\zeta) .
$$

Upon writing the above expression, it has been used that the minimum values of $\left|\boldsymbol{r}-\boldsymbol{r}_{1}\right|$, for given values of $z$ and $z_{1}$, is $\left|z-z_{1}\right|$. Carrying out the integration over $\zeta$, one gets

$$
H^{c}(t)=\frac{\pi}{2} \int d x \int d y \int_{\sigma / 2}^{h-\sigma / 2} d z \int_{\sigma / 2}^{h-\sigma / 2} d z_{1} n(\boldsymbol{r}, t) n\left(\boldsymbol{r}_{1}, t\right)\left[\sigma^{2}-\left(z-z_{1}\right)^{2}\right],
$$

and from here

$$
\frac{\partial H^{c}(t)}{\partial t}=-I(t)
$$

with $I(t)$ given again by Eq. (39). In the derivation of the above result, the time derivative on the right-hand side has been computed by means of the continuity equation,

$$
\frac{\partial}{\partial t} n(\boldsymbol{r}, t)=-\frac{\partial}{\partial \boldsymbol{r}}[n(\boldsymbol{r}, t) \boldsymbol{u}(\boldsymbol{r}, t)],
$$

which follows directly from the kinetic Eq. (25) by considering the equation for the first velocity moment. Moreover, an integration by parts has been performed. Combination of Eqs. (26), (38), and (42) yields

$$
\frac{\partial H(t)}{\partial t} \leqslant 0
$$

The inequality used when going from Eq. (34) to Eq. (35) implies that the equality sign in the above relation happens if and only if

$$
f\left(\boldsymbol{r}, \boldsymbol{v}^{\prime}, t\right) f\left(\boldsymbol{r}_{1}, \boldsymbol{v}_{1}^{\prime}, t\right)=f(\boldsymbol{r}, \boldsymbol{v}, t) f\left(\boldsymbol{r}_{1}, \boldsymbol{v}_{1}, t\right) .
$$

In principle, from Eq. (34) it could be thought that this condition is only required for velocities $v$ and $v_{1}$ such that $\boldsymbol{g} \cdot \widehat{\boldsymbol{\sigma}}<0$, but notice that $\boldsymbol{v}$ and $\boldsymbol{v}_{1}$ can be also interpreted as the postcollisional velocities for particles colliding with velocities $\boldsymbol{v}^{\prime}$ and $\boldsymbol{v}_{1}^{\prime}$, verifying these new precollisional velocities that $\boldsymbol{g}^{\prime} \cdot \widehat{\boldsymbol{\sigma}}>0$, for the same given $\widehat{\boldsymbol{\sigma}}$.

Let us assume, on a physical basis, that the total number of particles is finite, i.e.,

$$
\int d \boldsymbol{r} \int d \boldsymbol{v} f(\boldsymbol{r}, \boldsymbol{v}, t)=N
$$

and that the total energy of the gas is bounded, i.e.,

$$
\int d \boldsymbol{r} \int d \boldsymbol{v} \frac{m v^{2}}{2} f(\boldsymbol{r}, \boldsymbol{v}, t)=E .
$$

Under these conditions, $H^{k}(t)$ is bounded from below [12]. Moreover, it is easy to verify that the configurational part $H^{c}$ as defined by Eq. (28), which depends on the local density only, is also bounded. Therefore, since $H(t)$ is bounded from below and it decreases monotonically in time, $H(t)$ must reach eventually a steady value, in which $f=\bar{f}$, such that Eq. (45) is verified, i.e.,

$$
\ln \bar{f}\left(\boldsymbol{r}, \boldsymbol{v}^{\prime}, t\right)+\ln \bar{f}\left(\boldsymbol{r}_{1}, \boldsymbol{v}_{1}^{\prime}, t\right)=\ln \bar{f}(\boldsymbol{r}, \boldsymbol{v}, t)+\ln \bar{f}\left(\boldsymbol{r}_{1}, \boldsymbol{v}_{1}, t\right) .
$$

This must be verified for all $x, y, z, z_{1}, \boldsymbol{v}, \boldsymbol{v}^{\prime}$, and $\varphi$. Let us write the relation in the form

$$
\ln \frac{\bar{f}\left(\boldsymbol{r}, \boldsymbol{v}^{\prime}, t\right)}{\bar{f}(\boldsymbol{r}, \boldsymbol{v}, t)}=-\ln \frac{\bar{f}\left(x, y, z_{1}, \boldsymbol{v}_{1}^{\prime}, t\right)}{\bar{f}\left(x, y, z_{1}, \boldsymbol{v}_{1}, t\right)} .
$$

The postcollisional velocities $\boldsymbol{v}^{\prime}$ and $\boldsymbol{v}_{1}^{\prime}$ are functions of $\boldsymbol{v}, \boldsymbol{v}_{1}$, $z-z_{1}$, and $\varphi$. The above equation expresses that, for given values of $\boldsymbol{v}, \boldsymbol{v}_{1}, \boldsymbol{v}^{\prime}$, and $\boldsymbol{v}_{1}^{\prime}$, the left-hand side does not depend on $z_{1}$, while under the same conditions the right-hand side does not depend on $z$. Consequently, none of the ratios at both sides of the relation depends either on $z$ or on $z_{1}$. Therefore, it must be

$$
\frac{\partial}{\partial z} \frac{\bar{f}\left(\boldsymbol{r}, \boldsymbol{v}^{\prime}, t\right)}{\bar{f}(\boldsymbol{r}, \boldsymbol{v}, t)}=0
$$

or

$$
\frac{\partial}{\partial z} \ln \bar{f}(\boldsymbol{r}, \boldsymbol{v}, t)=\frac{\partial}{\partial z} \ln \bar{f}\left(\boldsymbol{r}, \boldsymbol{v}^{\prime}, t\right) .
$$

The velocity $\boldsymbol{v}^{\prime}$ is a continuous function of $\boldsymbol{v}, \boldsymbol{v}_{1}, \varphi$, and $z-z_{1}$. Therefore, if it is assumed that the one-particle distribution function $\bar{f}(\boldsymbol{r}, \boldsymbol{v}, t)$ is a continuous function of $\boldsymbol{v}, \mathrm{Eq}$. (51) implies that

$$
\frac{\partial}{\partial z} \ln \bar{f}(\boldsymbol{r}, \boldsymbol{v}, t)=A(\boldsymbol{r}, t)
$$

and integration yields

$$
\ln \bar{f}(\boldsymbol{r}, \boldsymbol{v}, t)=\Phi(\boldsymbol{r}, t)+\Phi_{0}(x, y, \boldsymbol{v}, t) .
$$

Substitution of this in Eq. (48) gives (the dependence on $x$ and $y$ is omitted)

$$
\Phi_{0}\left(\boldsymbol{v}^{\prime}, t\right)+\Phi_{0}\left(\boldsymbol{v}_{1}^{\prime}, t\right)=\Phi_{0}(\boldsymbol{v}, t)+\Phi_{0}\left(\boldsymbol{v}_{1}, t\right) .
$$


This is the familiar condition characterizing collision invariants, and its solution is

$$
\Phi_{0}(\boldsymbol{v}, t)=\boldsymbol{a} \cdot \boldsymbol{v}+b v^{2}+c,
$$

where $\boldsymbol{a}, b$, and $c$ do not depend on $\boldsymbol{v}$ or $z$. Combining Eqs. (53) and (55), it is found

$$
\ln \bar{f}(\boldsymbol{r}, \boldsymbol{v}, t)=\Phi(\boldsymbol{r}, t)+\boldsymbol{a}(x, y, t) \cdot \boldsymbol{v}+b(x, y, t) v^{2}+c(x, y, t)
$$

or

$$
\bar{f}=n\left(\frac{m}{2 \pi k_{B} T}\right)^{3 / 2} \exp -\frac{m(\boldsymbol{v}-\boldsymbol{u})^{2}}{2 k_{B} T},
$$

where $k_{B}$ is the Boltzmann constant. The new parameters $n, \boldsymbol{u}$, and $T$, that can be functions of $x, y$, and $t$, are related with $\Phi$, $a, b$, and $c$. The explicit form of the relations can be obtained by direct comparison, but they will be not relevant here. The advantage of writing $\bar{f}$ in the form of Eq. (57) is that $n, \boldsymbol{u}$, and $T$ are easily identified as the local number density, local field velocity, and local temperature, respectively. The latter is defined as

$$
\frac{3}{2} n(\boldsymbol{r}, t) k_{B} T(\boldsymbol{r}, t) \equiv \frac{m}{2} \int d \boldsymbol{v}[\boldsymbol{v}-\boldsymbol{u}(\boldsymbol{r}, t)]^{2} f(\boldsymbol{r}, \boldsymbol{v}, t) .
$$

It is worth emphasizing a main difference between $\bar{f}$ given by Eq. (57) and the local equilibrium distribution function canceling the Boltzmann collison term of a nonconfined system. In the latter, $\boldsymbol{u}$ and $T$ can be local quantities depending on $\boldsymbol{r}$, while in Eq. (57) they cannot depend on the $z$ coordinate. On the other hand, they can depend on time. It still remains to require $\bar{f}$ to satisfy the kinetic Eq. (25) with the corresponding boundary conditions. When this is done, it follows that the only solution corresponds to vanishing $\boldsymbol{u}$, constant and uniform $T$, and a time-independent density profile depending only on the $z$ coordinate. More specifically, the steady solution of the equation, always reached in the long time limit, is

$$
f_{s t}(\boldsymbol{r}, \boldsymbol{v})=n(z) \varphi_{\mathrm{MB}}(v),
$$

where $\varphi_{\mathrm{MB}}(v)$ is the Maxwellian velocity distribution,

$$
\varphi_{\mathrm{MB}}(v) \equiv\left(\frac{m}{2 \pi k_{B} T}\right)^{3 / 2} e^{-\frac{m v^{2}}{2 k_{B} T}}
$$

and

$$
n(z)=\frac{N}{A b} \exp \left[a\left(z-\frac{h}{2}\right)^{2}\right] .
$$

Here $A$ is the area of each of the two parallel plates, $a \equiv$ $\pi N / A$, and

$$
b \equiv \sqrt{\frac{\pi}{a}} \operatorname{erfi}\left[\frac{\sqrt{a}}{2}(h-\sigma)\right] .
$$

Here erfi $(x)$ is the imaginary error function defined as

$$
\operatorname{erfi}(x) \equiv \pi^{-1 / 2} \int_{-x}^{x} d y e^{y^{2}}
$$

An outline of the derivation of Eq. (59) is provided in Appendix B. It is inspired in the analysis carried out for the usual Boltzmann equation [19]. The stationary density profile in Eq. (61) agrees with the low density limit of the expression given by Schmidt and Löwen [6], when keeping terms up to second order in both results. In the context of the kinetic Boltzmann equation considered in this paper, the vertical inhomogeneity follows from the $z$ dependence of the collision term, which in turn is a consequence of the confinement of the system. The latter limits the possible collision angles, the allowed range being a function of the $z$ coordinate of the considered target particle. The accuracy of the predicted density profile has been checked by means of molecular dynamics simulations $[15,20]$. Also, other theoretical predictions for the stationary state, like the entropy or some components of the pressure tensor, have been analyzed.

\section{EVOLUTION EQUATIONS FOR THE TEMPERATURE PARAMETERS}

In the previous section, it has been shown that the kinetic equation leads to the existence of a unique steady state whose properties are in full agreement with previous predictions derived by means of equilibrium statistical mechanics theories. In this section, the macroscopic relaxation of the system towards equilibrium in a simple situation will be addressed. We will consider states in which the system is homogeneous in the horizontal $x-y$ plane, and define average properties in the $z$ direction. They will be denoted by means of an overline on the corresponding symbol. For the number density it is

$$
\bar{n} \equiv \frac{1}{h-\sigma} \int_{\sigma / 2}^{h-\sigma / 2} d z n(z)=\frac{N}{A(h-\sigma)} \equiv n_{0},
$$

i.e., it agrees with average number density in the system. Averaged vertical and horizontal temperature parameters are defined by

$$
n_{0} k_{B} \bar{T}_{z}(t) \equiv \frac{m}{h-\sigma} \int_{\sigma / 2}^{h-\sigma / 2} d z \int d \boldsymbol{v} v_{z}^{2} f(z, \boldsymbol{v}, t)
$$

and

$$
n_{0} k_{B} \bar{T}_{x y}(t) \equiv \frac{m}{2(h-\sigma)} \int_{\sigma / 2}^{h-\sigma / 2} d z \int d \boldsymbol{v}\left(v_{x}^{2}+v_{y}^{2}\right) f(z, v, t),
$$

respectively. It has been implicitly assumed that there is no macroscopic velocity flow, i.e., $\boldsymbol{u}=0$. Of course, a global scalar temperature $\bar{T}(t)$ can be identified as

$$
\bar{T}(t)=\frac{\bar{T}_{z}+2 \bar{T}_{x y}}{3} .
$$

From the kinetic Eq. (25) it follows that, for elastic boundaries so that there is no energy flux through them, it is

$$
\begin{aligned}
n_{0} k_{B} \frac{\partial \bar{T}_{z}(t)}{\partial t}=\frac{m}{h-\sigma} & \int_{\sigma / 2}^{h-\sigma / 2} d z \int d \boldsymbol{v} v_{z}^{2} J[z, \boldsymbol{v} \mid f], \\
n_{0} k_{B} \frac{\partial \bar{T}_{x y}(t)}{\partial t}= & \frac{m}{2(h-\sigma)} \int_{\sigma / 2}^{h-\sigma / 2} d z \\
& \times \int d \boldsymbol{v}\left(v_{x}^{2}+v_{y}^{2}\right) J[z, \boldsymbol{v} \mid f] .
\end{aligned}
$$


These equations lead to

$$
\frac{\partial \bar{T}(t)}{\partial t}=0
$$

as it should be by energy conservation. To evaluate the integrals on the right-hand side of Eqs. (68) and (69), a local-equilibrium-like approximation will be employed. The one-particle distribution function will be written as

$$
f(z, \boldsymbol{v}, t) \approx \frac{n_{0}}{\pi^{3 / 2} \omega_{x y}^{2}(t) \omega_{z}(t)} e^{-\frac{v_{x}^{2}+v_{y}^{2}}{\omega_{x y}^{2}(t)}-\frac{v_{z}^{2}}{\omega_{z}^{2}(t)}},
$$

where

$$
\begin{aligned}
\omega_{x y}^{2}(t) & \equiv \frac{2 k_{B} T_{x y}(t)}{m}, \\
\omega_{z}^{2}(t) & \equiv \frac{2 k_{B} T_{z}(t)}{m} .
\end{aligned}
$$

When Eq. (71) is substituted into the right-hand side of Eqs. (68) and (69), the evaluation of the integrals is still rather involved due to the $z$-dependence of the collision rate [see Eq. (9)]. Nevertheless, the calculations, although tedious, can be easily done by means of a symbolic computation program. Here results using Mathematica [21] will be reported. For the sake of simplicity an expansion in the parameter,

$$
e \equiv \frac{h-\sigma}{\sigma}
$$

has been carried out, keeping terms up to $e^{3}$ order, which is the first nontrivial one. The resulting equations read

$$
\begin{aligned}
& k_{B} \frac{\partial \bar{T}_{z}(t)}{\partial t}=\frac{2 m}{3}\left(\frac{\pi}{2}\right)^{1 / 2} n_{0} \sigma^{2} e^{3} \omega_{x y}\left(\omega_{x y}^{2}-\omega_{z}^{2}\right), \\
& k_{B} \frac{\partial \bar{T}_{x y}(t)}{\partial t}=\frac{m}{3}\left(\frac{\pi}{2}\right)^{1 / 2} n_{0} \sigma^{2} e^{3} \omega_{x y}\left(\omega_{z}^{2}-\omega_{x y}^{2}\right) .
\end{aligned}
$$

Of course, the latter equation is redundant, since it follows directly from Eq. (75) and the energy conservation, Eq. (70). According to the above equations, the rate of energy interchange between the horizontal and the vertical degrees of freedom is linear in the difference of the temperature parameters. A similar relation was considered by Khain and Aranson [22] on the basis of more qualitative reasonings. Also notice that the only stationary temperatures are $\bar{T}_{x y}=\bar{T}_{z}=$ $\bar{T}$, consistently with the $H$ theorem derived in Sec. III.

To investigate the accuracy of the theoretical prediction given by the above equations, MD simulations have been performed. The results to be reported below correspond to a system of 500 hard spheres confined between two hard plates separated a distance $h=1.5 \sigma$. Periodic boundary conditions are used in the $x$ and $y$ directions. The size of the unit square cell in the horizontal plane has been chosen such that the average number of particles density is $n_{0}=0.06 \sigma^{-3}$. It is worth insisting in the definition of $n_{0}$ given by Eq. (64), in which only the volume between the two plates that is accessible to the center of the hard spheres is considered. Of course, this is just a matter of definition and other options are equally acceptable [6]. The initial temperatures were chosen homogeneous and such that $T_{z}(0)=3 T_{x y}(0)$, and the initial marginal velocity distribution of the components of the

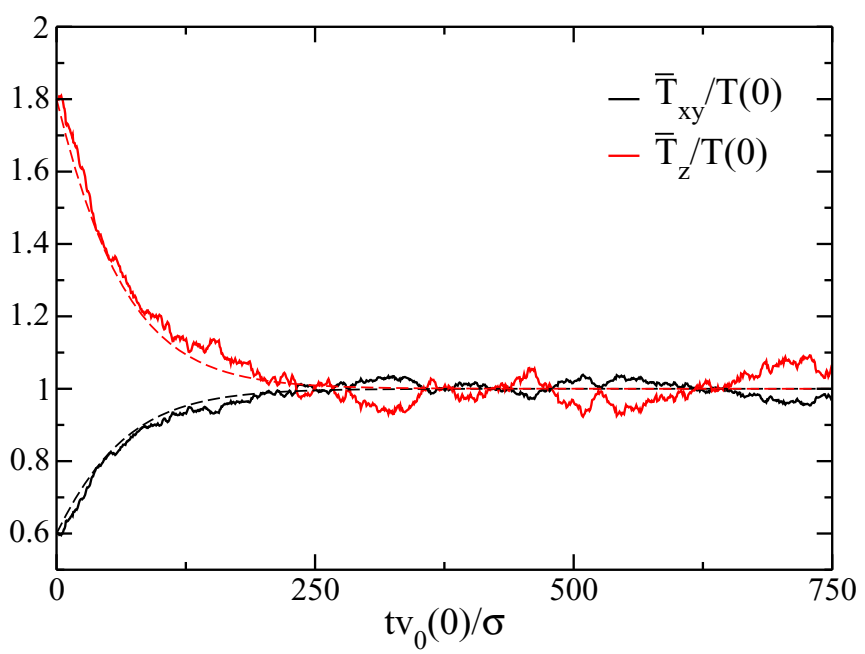

FIG. 2. Decay of the averaged vertical, $\bar{T}_{z}$, and horizontal, $\bar{T}_{x y}$, temperature parameters toward their equal steady values in a confined system that is homogeneous in the horizontal $x-y$ plane. Time is measured in the dimensionless units indicated in the label, with $v_{0} \equiv \sqrt{2 k_{B} T(0) / m}$. The solid lines are MD simulation results while the dashed lines are the theoretical predictions given by Eqs. (75) and (76). Moreover, the two (red) upper lines correspond to the vertical temperature parameter and the two (black) lower lines to the horizontal temperature parameter.

velocity were Gaussian. In Fig. 2, the simulation results are compared with the numerical solutions of Eqs. (75) and (76). A quite good agreement is observed, especially taking into account that the parameter $e$ defined in Eq. (74) is 0.5, that is not very small. In particular, both temperature parameters converge monotonically to the steady global temperature value.

\section{SUMMARY AND FINAL COMMENTS}

In this paper, the Boltzmann kinetic equation for a quasitwo-dimensional system of hard spheres confined between two parallel hard plates has been derived. Moreover, an entropy Lyapunov function for the equation has been identified, and the stationary one-particle distribution function has been determined. It is a Gaussian in velocity space, as expected, having a nonuniform density in the direction perpendicular to the confining walls. The density profile is consistent with the results obtained by using equilibrium statistical mechanics methods and, in particular, functional density theory.

To get some information about the mechanisms for which energy is interchanged between the horizontal and vertical degrees of freedom, the time evolution of the temperature parameters associated with them has been analyzed in a system that is homogeneous in the horizontal plane. Using some kind of local equilibrium approximation the temperatures are shown to obey nonlinear ordinary first order differential equations, implying a monotonic convergence of both temperature parameters toward the common steady value. Molecular dynamics simulations have been performed and a good agreement with the theoretical predictions has been found.

The next natural step is to consider nonequilibrium situations exhibiting gradients in the horizontal direction, and 
try to derive macroscopic hydrodynamiclike equations for the time evolution of the macroscopic fields. This can be done by using a modification of the well-established ChapmanEnskog algorithm, adopted for the present case in which the equilibrium reference state is inhomogeneous, or by focusing directly on particular non-equilibrium steady states whose symmetry allows for important simplifications of the kinetic equation. Work along these lines is being carried out.

As said in the Introduction, the study of a confined quasitwo-dimensional system of hard spheres has been prompted and stimulated by the experimental results obtained with fluidized systems of macroscopic glass or iron spheres, i.e., in granular gases. The kinetic Boltzmann equation considered in this paper can be easily extended for inelastic hard spheres, similarly to what has been done for the ordinary Boltzmann equation [23]. The only needed modification refers to the collision rule. Then, the reference state must be identified. It should be a generalization of the homogeneous cooling state of bulk granular fluids [24]. Afterwards, the derivation of hydrodynamiclike equations can be addressed. It is at this point when the analysis carried out here becomes relevant, since a necessary and key ingredient in these equations must be the mechanism of transference of energy from the vibrating walls to the horizontal degrees of freedom of the fluid. Of course, it is needed to adapt the present theory to the case of inelastic collisions, but again this is straightforward. Finally, the constitutive relations for the several fluxes can be derived by means of the linear theory [25,26] or by employing a generalized Chapmann-Enskog algorithm [27].

\section{ACKNOWLEDGMENTS}

This research was supported by the Ministerio de Economía y Competitividad (Spain) through Grant No. FIS2014-53808-P (partially financed by FEDER funds).

\section{APPENDIX A: THE WALLS COLLISION TERMS IN THE KINETIC EQUATION}

In this Appendix, the expressions of the contributions originated by the collisions of the particles with the hard walls $\bar{T}_{W} f$ to the kinetic Eq. (3) will be derived. Consider the hard wall located at $z=0$ and write

$$
\begin{aligned}
& \{[f(\boldsymbol{r}, \boldsymbol{v}, t+\delta t)-f(\boldsymbol{r}, \boldsymbol{v}, t)] d \boldsymbol{r} d \boldsymbol{v}\}_{\text {wall }} \\
& \quad=\Gamma_{W}^{(0)}=\Gamma_{W^{+}}^{(0)}-\Gamma_{W^{-}}^{(0)} .
\end{aligned}
$$

The term $\Gamma_{W^{-}}$is the number of particles in the volume element $d \boldsymbol{r}$ around $\boldsymbol{r}$ and with velocity between $\boldsymbol{v}$ and $\boldsymbol{v}+d \boldsymbol{v}$ changing their velocities as a consequence of a collision with the wall in the time interval between $t$ and $t+\delta t$. On the other hand, $\Gamma_{W^{+}}$is the number of particles inside $d \boldsymbol{r}$ that get a velocity between $\boldsymbol{v}$ and $\boldsymbol{v}+d \boldsymbol{v}$ because of a collision with the wall in the time interval $(t, t+\delta t)$. From these definitions, using elementary kinetic theory arguments based on the collision cylinder construction, one gets

$$
\begin{gathered}
\Gamma_{W^{-}}^{(0)}=\Theta\left(-v_{z}\right)\left|v_{z}\right| \delta\left(z-\frac{\sigma}{2}\right) f(\boldsymbol{r}, \boldsymbol{v}, t) d \boldsymbol{r} d \boldsymbol{v} \delta t, \\
\Gamma_{W^{+}}^{(0)}=\Theta\left(-v_{z}^{*}\right)\left|v_{z}^{*}\right| \delta\left(z-\frac{\sigma}{2}\right) f\left(\boldsymbol{r}, \boldsymbol{v}^{*}, t\right) d \boldsymbol{r} d \boldsymbol{v}^{*} \delta t,
\end{gathered}
$$

where $v^{*}$ is the velocity that a particle must have before colliding with the wall to get a velocity $v$ after the collision, i.e.,

$$
\boldsymbol{v}^{*}=\boldsymbol{v}-2 v_{z} \boldsymbol{e}_{z}
$$

Therefore, $d \boldsymbol{v}^{*}=d \boldsymbol{v}, \Theta\left(v_{z}^{*}\right)=\Theta\left(-v_{z}\right)$, and $\left|v_{z}^{*}\right|=\left|v_{z}\right|$. Using these relations, $\Gamma_{W^{+}}^{(0)}$ can be expressed in terms of $v$ instead of $\boldsymbol{v}^{*}$, and substitution of the result and Eq. (A2) into Eq. (A1) yields

$$
\begin{aligned}
\Gamma_{W}^{(0)}= & \delta\left(z-\frac{\sigma}{2}\right) v_{z}\left[\Theta\left(v_{z}\right) f\left(\boldsymbol{r}, \boldsymbol{v}-2 v_{z} \boldsymbol{e}_{z}, t\right)\right. \\
& \left.+\Theta\left(-v_{z}\right) f(\boldsymbol{r}, \boldsymbol{v}, t)\right] d \boldsymbol{r} d \boldsymbol{v} \delta t
\end{aligned}
$$

From this result, the expression of $\bar{T}_{W}^{(0)} f$ given in Eq. (16) follows directly. Repeating the same argument for the wall located at $z=h$ leads to Eq. (17). The same results can be derived by considering the collision term for two hard spheres of different diameters and letting one of the diameters become infinite.

\section{APPENDIX B: THE STATIONARY DISTRIBUTION FUNCTION}

The aim of this Appendix is to sketch the derivation of Eq. (59) from Eq. (57). To begin with, let us rewrite the former as

$$
\bar{f}=g \exp \left[-\frac{m}{2 k_{B} T}\left(v^{2}-2 \boldsymbol{u} \cdot \boldsymbol{v}\right)\right],
$$

with

$$
g \equiv n\left(\frac{m}{2 \pi k_{B} T}\right)^{3 / 2} \exp -\frac{m u^{2}}{2 k_{B} T} .
$$

Notice that $g$ does not depend on the velocity $\boldsymbol{v}$. When Eq. (B1) is substituted into Eq. (25), the result is a sum of terms, each of them being $\exp \left[-\frac{m}{2 k_{B} T}\left(v^{2}-2 \boldsymbol{u} \cdot \boldsymbol{v}\right)\right]$ times a polynomial that is up to third degree in the components of $\boldsymbol{v}$. The equation must hold identically in $\boldsymbol{v}$, so each of the coefficients of a velocity monomial is required to vanish identically. The term that is independent from $\boldsymbol{v}$ leads to the equation

$$
\frac{\partial g}{\partial t}=0
$$

and the vanishing of the cubic terms requires

$$
\nabla T=0 .
$$

The remaining two equations, corresponding to linear and quadratic terms are

$$
\begin{gathered}
\nabla \ln g+\frac{\partial}{\partial t}\left(\frac{m \boldsymbol{u}}{k_{B} T}\right)=2 \pi \boldsymbol{e}_{z} \int_{\sigma / 2}^{h-\sigma / 2} d z_{1} n\left(\boldsymbol{r}_{1}\right)\left(z-z_{1}\right), \\
\frac{\partial \ln T}{\partial t} \delta_{i j}+\frac{\partial u_{i}}{\partial r_{j}}+\frac{\partial u_{j}}{\partial r_{i}}=0 .
\end{gathered}
$$

The solution of the latter equation is

$$
\boldsymbol{u}(\boldsymbol{r}, t)=\omega(t) \times \boldsymbol{r}-\frac{1}{4} \frac{\partial \ln T}{\partial t} \boldsymbol{r}+\boldsymbol{u}_{0}(t),
$$

where $\boldsymbol{u}_{0}(t)$ and $\boldsymbol{\omega}(t)$ are homogeneous time dependent vectors. This can be verified by direct substitution. Equation (B7) 
shows that the general $\boldsymbol{u}$ is a combination of a time dependent uniform velocity, uniform rotation, and uniform dilation. For the system we are considering, with hard horizontal plates and periodic boundary conditions in the $x$ and $y$ directions, the lack of an axis of symmetry and momentum conservation lead to

$$
\omega=\boldsymbol{u}_{0}=0
$$

and

$$
\frac{\partial T}{\partial t}=0
$$

so that $\boldsymbol{u}=0$. Use of the above results into Eq. (B5) yields

$$
\begin{gathered}
\frac{\partial n}{\partial x}=\frac{\partial n}{\partial y}=0 \\
\frac{\partial n(z)}{\partial z}=2 \pi n(z) \int_{\sigma / 2}^{h-\sigma / 2} d z_{1} n\left(z_{1}\right)\left(z-z_{1}\right) .
\end{gathered}
$$

The solution of this differential equation is just Eq. (61). This completes the derivation of Eq. (59) in the main text.
[1] Molecular Dynamics in Restricted Geometries, edited by J. Klafter and J. M. Drake (John Wiley, New York, 1989).

[2] C. Alba-Simionesco, B. Coasne, G. Dosseh, G. Dudziak, K. E. Gubbins, R. Radhakrishnan, and M. Sliwinska-Bartkowiak, J. Phys.: Condens. Matter 18, R15 (2006).

[3] S. Dietrich and A. Haase, Phys. Rep. 260, 1 (1995).

[4] R. Evans, J. Phys.: Condens. Matter 2, 8989 (1990).

[5] T. Franosch, S. Lang, and R. Schilling, Phys. Rev. Lett. 109, 240601 (2012).

[6] M. Schmidt and H. Löwen, Phys. Rev. Lett. 76, 4552 (1996); Phys. Rev. E 55, 7228 (1997).

[7] P. Melby, F. V. Reyes, A. Prevost, R. Robertson, P. Kumar, D. A. Egolf, and J. S. Urbach, J. Phys.: Condens. Matter 17, S2689 (2005).

[8] J. S. Olafsen and J. S. Urbach, Phys. Rev. Lett. 95, 098002 (2005).

[9] M. G. Clerc, P. Cordero, J. Dunstan, K. Huff, N. Mújica, D. Risso, and G. Varas, Nat. Phys. 4, 249 (2008).

[10] I. Goldhirsch, Annu. Rev. Fluid Mech. 35, 267 (2003).

[11] J. R. Dorfman and H. van Beijeren, in Statistical Mechanics, Part B, edited by B. J. Berne (Plenum Press, New York, 1977).

[12] P. Résibois and M. de Leener, Classical Kinetic Theoty of Fluids (John Wiley and Sons, New York, 1977).
[13] J. A. McLennan, Introduction to Non-equilibrium Statistical Mechanics (Prentice Hall, New Jersey, 1989).

[14] C. Cercignani, Theory and Application of the Boltzmann Equation (Scottish Academic Press, Edinburgh, 1975).

[15] J. J. Brey, P. Maynar, and M. I. García de Soria, Phys. Rev. E 94, 040103(R) (2016).

[16] J. Kestin and J. R. Dorfman, A Course in Statistical Thermodynamics (Academic Press, New York, 1971).

[17] P. Résibois, Phys. Rev. Lett. 40, 1409 (1978).

[18] P. Résibois, J. Stat. Phys. 19, 593 (1978).

[19] G. E. Uhlenbeck and G. W. Ford, Lectures in Statistical Mechanics (American Mathematical Society, Providence, 1963)

[20] J. J. Brey, V. Buzón, M. I. García de Soria, and P. Maynar, Entropy 19, 68 (2017).

[21] Wolfram Research Inc., Mathematica (Wolfram Reserach Inc., Champaign, Illinois, 2016).

[22] E. Khain and I. S. Aranson, Phys. Rev. E 84, 031308 (2011).

[23] A. Goldstein and M. Shapiro, J. Fluid. Mech. 282, 75 (1995).

[24] P. K. Haff, J. Fluid Mech. 134, 401 (1983).

[25] J. W. Dufty and J. J. Brey, J. Stat. Phys. 109, 433 (2002).

[26] J. J. Brey and M. J. Ruiz-Montero, Phys. Rev. E 70, 051301 (2004).

[27] J. J. Brey, J. W. Dufty, C. S. Kim, and A. Santos, Phys. Rev. E 58, 4638 (1998). 